(Aus der Königl. Frauenklinik in Dresden.)

\title{
Die Perforation des lebensfrischen und absterbenden Kindes 1892-1906.
}

Von

\section{Meissner,}

Assistenzarzt.

Je mehr im Laufe der Jahre in der Dresdener Klinik zur Rettung des Kindes der Kaiserschnitt ausgeführt wurde, um so grösser war die Zurückdrängung der Perforation des lebenden Kindes.

Und doch kamen in jedem Jahre ein oder mehrere Fälle noch vor, in denen auch bei noch lebensfrischem oder bereits absterbendem Kinde im Interesse der Mütter die Perforation die einzige richtige Maassnahme war.

Bei der grossen Begeisterung, mit welcher heut zu Tage einzelne Geburtshelfer bezw. Kliniken von der Perforation des lebenden Kindes nichts mehr wissen wollen, erscheint es angebracht, unser Material zu mustern und offen zur Beurtheilung vorzulegen. Es sollen die vorgekommenen Fehler in der Diagnostik, in der Anzeigestellung und Technik nicht verschwiegen werden, um dann angeben zu können, von welchen Gesichtspunkten aus fernerhin die Perforation des lebenden Kindes zu beurtheilen sein wird.

Von jeher ist in unserer Klinik der Grundsatz befolgt worden, jeden Fall von engem Becken von Anfang an nach seinen Eigenthümlichkeiten mit Unterstützung durch die Anamnese zu beurtheilen.

Obenan stand von jeher und heute noch das Bestreben, für Mutter und Kind den denkbar besten Ausgang zu wählen. In erster Linie wird stets erwogen, den Fall durch spontane Geburt endigen zu lassen. Erscheint dies unmöglich, nun so haben diejenigen 
666 Meissner, Perforation des lebensfrischen und absterbenden Kindes.

Operationen einzutreten, welche im einzelnen Falle vor der Wissenschaft begründet erscheinen. Der Wahl der Operation muss aber stets eine sorgfältige Abwägung der für die Mutter erwachsenden etwa grösseren Gefahr vorausgehen.

Dieser Gedankengang liegt auch denjenigen Fällen zu Grunde, welche wir jetzt betrachten wollen, den in den Jahren 1892 bis mit 1906 bei uns ausgeführten 57 Perforationen des lebenden und 112 Perforationen des absterbenden Kindes.

Sie vertheilen sich auf $14 \frac{1}{2}$ Jahre und auf eine Gesammtzahl von 29725 Geburten. Es kommen also auf ein Jahr von durchschnittlich 2123 Geburten ca. $4=0,2$ pCt. Perforationen des lebenden und ca. 8 Perforationen des absterbenden Kindes. Am seltensten machte sich die Enthirnung des lebenden Kindes 1902 nothwendig: 1 mal unter 2262 Entbindungen.

\begin{tabular}{l|r|r|r|r}
\hline & $\begin{array}{c}\text { I. } \\
\text { Jebendes } \\
\text { Kind }\end{array}$ & $\begin{array}{c}\text { II. } \\
\text { ab- } \\
\text { sterbendes } \\
\text { Kind }\end{array}$ & $\begin{array}{c}\text { Geburten- } \\
\text { zahl }\end{array}$ & $\begin{array}{c}\text { Gesammt- } \\
\text { zahl }\end{array}$ \\
\hline 1892 & 2 & 2 & 755 & 4 \\
1893 & 3 & 10 & 1670 & 13 \\
1894 & 5 & 6 & 1758 & 11 \\
1895 & 4 & 10 & 1847 & 14 \\
1896 & 2 & 12 & 2029 & 14 \\
1897 & 4 & 11 & 2100 & 15 \\
1898 & 7 & 9 & 2282 & 16 \\
1899 & 3 & 6 & 2087 & 9 \\
1900 & 5 & 6 & 2100 & 11 \\
1901 & 4 & 7 & 2167 & 11 \\
1902 & 1 & 6 & 2262 & 7 \\
1903 & 3 & 5 & 2170 & 8 \\
1904 & 2 & 9 & 2375 & 11 \\
1905 & 7 & 4 & 2057 & 11 \\
1906 & 5 & 9 & 2066 & 14 \\
\hline & 57 & 112 & 29725 & 169 \\
& & &
\end{tabular}

I. Perforation des lebenden Kindes.

Von den 57 Frauen hatten 8 ein fast normales, 49 ein enges Becken und zwar

2 ein plattes,

7 "platt rhachitisches,

19 " allgemein verengtes,

$19 " \quad$ verengt platt rhachitisches und

$2 n$ n und schräg verengtes Becken. 
Bei diesen 49 Frauen erreichte die

Conjugata vera bis $7,6 \mathrm{~cm} 1 \mathrm{mal}$,

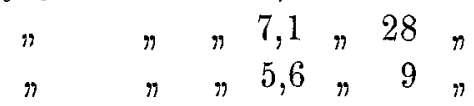

Bei den übrig bleibenden 11 engen Becken ist die Vera im Geburtsbericht nicht angegeben und zwar aus folgenden Gründen: 2 mal konnte die Diagonalis wegen eingekeilten Kopfes, 3 mal wegen Missgeburt nicht gemessen werden. 3 mal hinderte die starke Kopfgeschwulst, 1 mal der in das Becken eingetretene Kopf, 1 mal grosse Unruhe bei Eklampsie die Messung; 1 mal handelte es sich um eine Frau mit Tuberc. laryngis, die spontan im VII. Monat in die Geburt eintrat. Hierbei war die Verengerung nicht so hochgradig, dass sie die Indication zur Perforation allein hätte abgeben können.

Von den 57 Müttern waren 36 Erst- und 21 Mehrgebärende.

Die Geburt hatte bei ihnen 12 Stunden bis 3 Tage und 13 Stunden gedauert, bis die Perforation sich nothwendig machte.

Vom Blasensprung bis zur Operation war 1 Stunde bis 5 Tage verstrichen.

Zehn von den 57 Frauen waren als Hausschwangere längere Zeit in der Klinik und 47 Frauen nur 1 Stunde bis zu 4 Tagen.

Die Kindeslagen vertheilen sich folgendermaassen:

\begin{tabular}{|c|c|c|c|}
\hline \multicolumn{3}{|c|}{21 mal erste } & \multirow{2}{*}{ Schädellage } \\
\hline 28 & $n$ & zweite & \\
\hline 2 & $\eta$ & erste & Stimnloco \\
\hline 2 & $n$ & zweite & surnage \\
\hline 1 & $n$ & erste & Gasiohtclar \\
\hline 1 & $n$ & zweite & islage \\
\hline
\end{tabular}

In 9 Fällen war der Kopf in das Becken eingetreten, 2 mal befand er sich in Beckenmitte, $3 \mathrm{mal}$ war er abgewichen und $43 \mathrm{mal}$ stand er noch beweglich über dem Beckeneingang, $7 \mathrm{mal}$ in Vorder- und $9 \mathrm{mal}$ in Hinterscheitelbeineinstellung.

Bei Beginn der Operation war der Muttermund eröffnet:

3 markstückgross. . . . in 5 Fällen

5

kleinhandtellergross . . . .

handtellergross . . . . . 11 ,

vollständig . . . . . $n^{21} n$ 
668 Meissner, Perforation des lebensfrischen und absterbenden Kindes.

In 4 Fällen wurde der Muttermund durch das Bossi'sche Instrument erweitert; es waren dies 3 Erstgebärende: eine mit Eklampsie, eine mit rigiden Weichtheilen in Folge Kolpitis granulosa, eine vom Arzt ausserhalb geschickte mit fötidem Fruchtwasser und Fieber 39,2; 100 - eine 37 jährige XI p. mit Eklampsie. In einem Falle von schwerer Eklampsie wurde der einmarkstückgrosse Muttermund durch Incisionen erweitert.

In 28 Fällen war ein Kolpeurynter, in 5 Fällen ein Metreurynter resp. Barnes'sche Blase zur Verwendung gekommen. In einem Falle (sehr schwere Nephritis), wo die künstliche Frühgeburt eingeleitet wurde, war eine Bougie eingeschoben worden.

In welchem Zustande kamen die Frauen in die Klinik?

Von den 47 Fraven, die schon in der Geburt befindlich die Hülfe der Klinik aufsuchten, waren bereits bei 8 Frauen vergebliche Entbindungsversuche ausserhalb angestellt worden, und zwar 4 mal durch die Zange bei zum Theil noch nicht vollständig erweitertem Muttermund, und 4 mal durch Zange und Wendung. Unter diesen wurden mit Fieber und schweren Verletzungen 3 Frauen eingeliefert; eine mit Verletzung der Scheide und der Schamlippen, die ein blutendes faustgrosses Hämatom der linken Labie zur Folge hatte; eine mit einem Riss im hinteren Scheidengewölbe, der bis in das Collum reichte; bei dieser Frau fanden sich zudem Gonokokken im Secret. Nach längerer Beobachtung ausserhalb und mehrfachen inneren Untersuchungen mit Fieber $(39,2)$ warden 5 Frauen der Klinik übergeben. Innerlich nicht untersucht wurden vom Arzt geschickt 7 Frauen, darunter 5 Eklampsien, eine mit uncompensirtem Herzfehler und bedrohlichen Staungserscheinungen (Dyspnoe u. s. w.); eine wegen hochgradiger Nephritis. Nach inneren Untersuchungen, die von Arzt und Hebamme ausserhalb erfolgt waren, wurden 14. Frauen hereingeschickt. Als Kuriosum und recht beweisend für das Blühen der Kurpfuscherei in Sachsen möge mitgetheilt werden, dass eine 21 jährige ledige Ip., die mit gesprungener Blase hier ankam, von 2 Naturheilkundigen mit nicht desinficirten Fingern untersucht worden war. Auf eigenen Antrieb, ohne vorher draussen untersucht worden zu sein, kamen nur 12 Frauen von 47 ( +10 Hausschwangere) oder 21 pCt.

In 9 Fällen gingen der Perforation hier in der Anstalt Entbindungsversuche zur Rettung des kindlichen Lebens voraus. In 1 Falle war der Zangenversuch, in 6 Fällen der Wendungsversuch, 
in 2 Fällen die atypische Zange vergeblich. 1 mal wurde durch Hofmeier'sche Impression, 6 mal durch Walcher'sche Hängelage versucht, den Kopf in das Becken zu bringen, 1 mal wurde Lageverbesserungsversuch vorgenommen.

Der Operation selbst ging in den meisten Fällen Narkose voraus, und zwar in 46 (von 57) mit Aether, 3 mal mit Chloroform, $1 \mathrm{mal}$ war es aus dem Geburtsbericht nicht ersichtlich; in 1 Falle bekam die Frau zur Schmerzlinderung 0,01 Morphium subcutan, da ihr in Folge eines uncompensirten Herzfehlers mit starker Dyspnoe eine allgemeine Narkose nicht zugemuthet werden konnte. In 6 Fällen wurde die Perforation absichtlich ohne Narkose ausgeführt, um den hier studirenden Aerzten zu zeigen, dass bei genügend erweitertem Muttermunde ohne nennenswerthe Schmerzen die Perforation mit nachfolgender Kranioklasie bei sehr schonungsvollem Vorgehen wohl möglich ist.

Ueber die Ausführung der Perforation möge nur erwähnt sein, dass sie stets mit der Siebold'schen Scheere und die Extraction fast ausnahmslos mit dem Kranioklasten erfolgte. Ein Kephalothryptor machte sich niemals nothwendig.

Aus welchen Gründen erfolgte nun die Perforation des lebenden Kindes?

Ordnen wir sie nach gewissen Gruppen, so kommen zunächst 7 Missbildungen in Betracht: 1 Meningocele und 6 Hydrocephalen; unter den letzteren hatte einer die ansehnliche Ausbildung von $59 \mathrm{~cm}$ Länge und $6000 \mathrm{~g}$ Schwere ohne Schädelinhalt.

Nun folgen 6 Fälle ron Eklampsie (3 Erst-, 2 Zweit- und 1 Elftgebärende), sämmtlich schwerster Art, mit 17-22 Anfällen ausserhalb der Klinik. Sie waren theils vielfach schon untersucht, theils litten sie an hochgradiger Nephritis; in einem Falle war das Kind sehr klein, in einem anderen war die Nabelschnur vorgefallen und pulsirte mangelhaft.

In allen diesen Fällen war die Perforation des lebenden. Kindes der für die Mütter noch schonendste Eingriff.

Die dritte Gruppe umfasst drei Franen mit absolut verengten Becken, bei denen aber die für die Sectio caesarea nothwendigen Vorbedingungen zur Rettung des kindlichen Lebens fehlten. Eine kam mit gesprungener Blase nach langer Bahnfahrt, draussen öfters von der Hebamme untersucht. Die zweite und dritte litten an Gonorrhoe und waren bereits innerlich viel untersucht worden. 
670 Theissner, Perforation des Iebensfrischen und absterbenden Kindes.

Diesen schliesst sich als 4. Gruppe die der acht Anoperirten an, welche der Klinik von ausserhalb überwiesen worden waren. Sie boten theils Tympanie, theils Tetanus uteri dar mit hohem Fieber und Contractionsring und waren den verschiedensten Entbindungsversuchen ausserhalb bereits ausgesetzt gewesen.

In drei weiteren Fällen (Gruppe 5) handelte es sich um schwere Allgemeinerkrankungen der Mutter. Eine im VII. Monat schwangere 31 jährige VIIlp. litt an Tuberculosis laryngis und einem tuberculösen Geschwür an der Portio. Man musste hier wegen absoluter Wehenschwäche das nicht lebensfähige Kind, $40 \mathrm{~cm}$ lang und $1260 \mathrm{~g}$ schwer, perforiren. Beim 2. Falle lag ein uncompensirter Herzfehler mit Folgeerscheinungen vor. Da hierbei plötzlich bedrohliche dyspnoische Zustände eintraten trotz prophylaktischer Digitalisgaben, musste man den im Becken stebenden Kopf perforiren. Beim 3. Fall musste die wegen Nephritis mit Folgeerscheinungen eingeleitete Frühgeburt durch Perforation beendet werden, da plötzlich hochgradige Dyspnoe auftrat.

In diesen 3 Fällen musste der erst 3 Markstüekgrosse Muttermund unter der Operation langsam gedehnt werden.

Als sechste Gruppe erfordern die 10 Frauen eingehende Erörterung, welche schon als Hausschwangere unter klinischer Beobachtung standen. Schaltet man zunächst 2 von ihnen aus, bei denen es sich, wie schon oben angeführt, um Hydrocephalus handelte, so kommen 8 Frauen in Betracht, bei denen im Geburtsverlauf unglückliche, vorher nicht zu erwartende Zufälle eintraten.

Im ersten Falle handelte es sich um eine 31 jährige IIp. mit linksseitig schräg verengtem Becken und einer Conjugata vera von $8 \mathrm{~cm}$, die aber wegen der schrägen Verengerung nicht in Betracht kommen konnte, IX. Monat der Schwangerschaft, kleines Kind, daher Spontanpartus beabsichtigt; die Vorbereitung der Weichtheile unterstützte man durch Einlegen eines Kolpeurynters. Unglücklicher Weise war die Fruchtblase vorzeitig gesprungen. Trotz Abwartens über 11/2 Tage trat der Kopf nicht ein; unteres Uterinsegment stark ausgedehnt; Contractionsring. Da die Frau entbunden werden musste, blieb nur die Perforation übrig; die Symphyseotomie, wie die atypische Zange schloss man wegen der schrägen Verengerung aus, zur Sectio caesarea waren die Vorbedingungen nicht mehr nach einer Geburtsdauer über $1 \frac{1}{2}$ Tag erfüllt. Die Hebotomie wurde damals noch nicht ausgeführt.

Im zweiten Falle war es eine $31 \mathrm{j}$. I p. mit allgemein ver- 
engtem Becken, Vera 9. Der Kopf bereits in das Becken eingetreten, rückte nicht tiefer; der Blasensprung war ohne vorherige Wehen vor 5 Tagen bereits erfolgt. Wegen Contractionsringes Zangenversuch; der Kopf trat trotz kräftiger Tractionen nicht tiefer. Auch hier blieb zu damaliger Zeit nichts übrig, als zu perforiren, die Symphyseotomie scheute man wegen kaum zu vermeidender Läsionen, weil es sich um eine alte I p. handelte. Jetzt würde die Hebotomie noch in Frage gekommen sein.

Im dritten Fall lag bei einer 28 jährigen 1 p. mit allgemein. verengtem Becken (Vera 8) der Kopf in Hinterscheitelbeineinstellung fest im Beckeneingang. Vergeblich war ein Lageverbesserungsversuch gemacht worden. Schliesslich musste wegen drohender Uterusruptur und sehr stürmischen Wehen zur Perforation geschritten werden. Zur Symphyseotomie entschloss man sich nicht wegen der schlechten Einstellung des Kopfes, von der Erfahrung ausgehend, dass selbst nach Durchtrennung des Beckenringes in dieser ungünstigen Einstellung der Kopf kaum mit der Zange hätte entwickelt werden können. Auch hier käme eher vielleicht die Hebotomie in Frage.

Die 4. Frau, 21 jährige I p., mit allgemein verengtem platt rhachitischem Becken und einer Vera von $81 / 2 \mathrm{~cm}$ bekam unter der Geburt 39,4-108; der Kopf blieb beweglich in Vorderscheitelbeineinstellung. Die schnelle Ausbildung eines Contractionsringes in Nabelhöhe liess wegen drohender Uterusruptur die Perforation des lebenden Kindes als das Richtigste erscheinen.

Fall 5: Bei der 17 jährigen I p. mit allgemein verengtem platt rhachitischem Becken (Vera $81 / 2$ ) und kräftigen Wehen war ebenfalls die spontane Geburt beabsichtigt. Der Kopf trat aber nicht ein, die Wehen wurden schwach und liessen zuletzt vollständig nach. Zuletzt kam ein Contractionsring hinzu. Der Kopf war gross und hart und hatte sich nicht configurirt. Von einem atypischen Zangenversuch konnte keine Rede sein. Zu einem grösseren, das Kind rettenden Eingriff war es nunmehr zu spät geworden.

In Fall 6 handelte es sich um eine 36 jährige VIII para mit allgemein verengt platt rhachitischem Becken (Vera 71/2), bei welcher, wenn der Kopf nicht eintreten sollte, die Wendung und Extraction ins Auge gefasst wurde. Beim Sprengen der Blase und Eingehen zur Wendung fühlt man aber bereits einen starren Contractionsring, der die Hand nicht passiren liess. Jetzt würde man in solchem Falle die Hebotomie angewandt haben. 
672 Meissner, Perforation des lebensfrischen und absterbenden Kindes.

In Fall 7 bot die 23 jährige I para eine von einer Ventrifixur berrührende Laparotomienarbe dar. Becken allgemein verengt. Muttermund sehr starr in Folge der bestehenden Kolpitis granulosa wurde durch Bossi schliesslich erweitert; da aber wegen Contractionsringes Gefahr drohte, die hohe Zange und alle anderen Operationen namentlich wegen des bestehendẻn Fluor und der vielen vorangegangenen inneren Untersuchungen bedenklich erschienen, so blieb nur die Perforation übrig.

Im 8. Falle (22 jährige I para mit allgemein verengt platt rhachitischem Becken (Vera $7 \frac{3}{4}$ ) und schwerer Nephritis) hatte sich, da der Kopf nicht eintrat, sehr bald ein Contractionsring ausgebildet, welcher schliesslich die Perforation nöthig machte.

Durchmustern wir diese acht Fälle nach dem jetzigen Stande unserer Erfahrungen, so wären heute in den Fällen 1, 2, 3, 6 und 8 die Kinder durch frühzeitige Hebotomie wohl rettbar gewesen. Nach dem damaligen Stand der Sache bleibt bei der nachträglichen Beurtheilung des Geburtsverlaufes und dem Zustande der Mütter für sie die Perforation immer noch der schonendste Eingriff.

Jedenfalls lehren auch diese Fälle, dass auch die exspectative Methode in der Geburtshilfe ihre natürlichen Grenzen hat. Was endlich die letzte (7.) Gruppe betrift, so traten 22 Kreissende bereits in der Geburt befindlich ein, waren ausserhalb vielfach untersucht und boten zum Theil recht bedrohliche Zustände dar. Hier kommen Tuberculose der Mütter, Tetania uteri, Fieber in der Geburt, Nephritis bei Herz- und Lungenerkrankung, Gonorrhoe, lang abgeflossenes Fruchtwasser und schon lange bestehender Contractionsring theils einzeln, theils in vielfacher Verbindung unter einander in Betracht, Zustände mit solcher Bedrohung für die Mutter, dass auch die Hebotomie nach dem heutigen Stand unseres Wissens als planvoll zur Rettung des Kindes nicht bezeichnet werden könnte.

Länge und Gewicht der perforirten Kinder, unter denen die Knaben mit 39:18 die Mädchen überwiegen, ersieht man aus folgender kleinen Tabelle:

Das grösste Kind hatte eine Länge von $59 \mathrm{~cm}$ und war $6000 \mathrm{~g}$ schwer (Hydroceph. permagnus), das kleinste $40 \mathrm{~cm}$ und $1260 \mathrm{~g}$ schwer.

Erwägt man, unter welchen zum Theil recht schwierigen Verbältnissen die Enthirnung und Extraction vorgenommen werden musste, so können die mannigfachen Verletzungen an Damm und. 


\begin{tabular}{c|r|r|r}
\hline Gewicht & Fälle & Länge & Fälle \\
\hline $1000-1500$ & 1 & $35-40$ & 1 \\
$1500-2000$ & 7 & $40-45$ & 3 \\
$2000-2500$ & 7 & $45-50$ & 11 \\
$2500-3000$ & 20 & über 50 & 39 \\
$3000-3500$ & 12 & gegeben & 3 \\
$3500-4000$ & 6 & - & - \\
4000 und mehr & 2 & - & - \\
aicht verzeichnet & & - & -
\end{tabular}

Scheide nicht Wunder nehmen. Rechnet man auch die allerkleinsten Zerreissungen, so müssen 13 Collumrisse verzeichnet werden, von denen ein, wie sich erst später herausstellte, hoch hinaufgehender Cervixriss zur Uterusruptur und zum Tode führte.

Die 45 jähr. VIII para hatte 6 mal spontan geboren (darunter 4 todte Kinder) und einmal abortirt. Nach verschiedenen, ausserbalb erfolgten Manipulationen wurde sie inficirt, mit 38,9 und $96 \mathrm{P}$. der Klinik äberwiesen. Vera $73 / 4 \mathrm{~cm}$. Stillstand der Geburt. Nichteintreten des Kopfes. Leider wurde vor dem hier erfolgten Wendungsversuch nicht festgestellt, ob bereits innere Verletzungen vorhanden waren. Jedenfalls wurde der ganz schonungsvolle Wendungsversuch sofort unterbrochen, als sich eine Dehnung des unteren Uterinsegmentes herausstellte, und die Geburt durch Perforation des lebenden Kindes geendet. (Knabe 52, 3600 !)

Bei der Einstellung nach der Geburt fand sich ein rechtsseitiger, hochhinaufragender Collumriss, welcher sofort genäht wurde. Tod am 3. Tage an Peritonitis. Complete Uterusruptur.

Mit Sicherheit war letztere nicht bei der Perforation erfolgt. ob beim Wendungsversuch, möge offen bleiben. Es fällt dann der Ausgang mit Bestimmtheit nicht der Perforation zur Last. Gleichwohl soll er auf Rechnung der Klinik verbleiben, wenn auch die Frau von aussen inficirt übernommen wurde, da von Anfang an die sofortige Perforation das Richtigste gewesen wäre.

Bei dem ausserordentlich unsauberen Material kann es nicht verwundern, dass das Wochenbett nur bei 32 Frauen (56 pCt.) ganz fieberfrei war. Die Anderen waren kürzere und längere Zeit krank an Portio- und Scheidenbelag, Parametritis, an complicirender Gonorrhoe and an Thrombose.

Gestorben sind von den 57 Frauen zwei; eine an Eclampsie naeh 22 Anfällen, die andere ist die eben erwähnte VIIIpara mit completer Uterusruptur, deren Tod die Klinik auf sich nimmt. Es 
674 Meissner, Perforation des lebensfrischen und absterbenden Kindes.

kommt dann ein mütterlicher Todesfall auf 56 Perforationen des lebenden Kindes $=1,7$ pCt.

Wer sich die Mühe nehmen würde, alle diese 57 Fälle in den Journalen genau zu studiren, würde zu der vollen Ueberzeugung kommen müssen, dass man einen weit höheren Procentsatz an Morbidität und Mortalität der Mutter zu verzeichnen haben würde, wenn man bei verschiedenen dieser Fälle zur Rettung des Kindes die Pubotomie ausgeführt hätte.

Darum ist es wohl begründet, ein Cavete auszusprechen.

Trotz der modernen operativen Geburtshülfe wird selbst in Kliniken die Perforation des lebenden Kindes nicht aus der Welt geschafft werden können. Immer wieder werden Fälle eingeliefert werden, in denen der Zustand der Mutter sehr bedenklich ist; andererseits werden auch bei von vornherein in der Klinik beobachteten Frauen Zufälle im Geburtsverlauf eintreten können, welche dem Geburtshelfer immer wieder das Wort mahnend vor die Seele führen müssen: "Erst die Mutter und dann das Kind!"

Nach diesen Darlegungen bedürfen aber auch die folgenden 112 Fälle einer näheren Begründung, auch wenn es sich bei ihnen allen mit Sicherheit um absterbende Kinder handelte. Es könnte ja doch immer gefragt werden: Liesse sich für das gefährdete Kind in dem oder jenem Falle nicht doch ein anderer operativer, selbst grösserer Eingriff unternehmen? Es mögen daher auch diese Fälle wieder in gewisse Gruppen getheilt sein, um den Ueberblick zu erleichtern und alle ermüdenden Einzelheiten wegzulassen.

Wenn aber eine grössere Zahl dieser Fälle doch ausführlicher mitgetheilt ist, so geschieht es hauptsächlich im Interesse des. praktischen Geburtshelfers, um ihm bei der Lectüre ein möglichst buntes Bild von den Verschiedenheiten und Schwierigkeiten der geburtshülflichen klinischen Ereignisse zu geben.

\section{Die Perforation des absterbenden Kindes.}

Haben wir bei einem Missverbältuiss zwischen Kopf und engem Becken oder bei bedrohlichem Zustande der Mutter ein absterbendes Kind vor uns, und ist eine rasche, die Mutter nicht schwer gefährdende Operation aller Voraussicht nach nicht im Stando, das absterbende Kind noch zu retten, so setzen wir heute noch das absterbende Kind dem todten gleich und perforiren. Wir opfern lieber 
dabei das bereits zweifelhafte Leben des Kindes, um wenigstens die Mutter mit grösster Sicherheit zu retten, als schliesslich ein todtes Kind zu erzielen nach schwerer Schädigung der Mutter.

Von den 112 Müttern, bei denen das absterbende Kind perforirt wurde, hatten 107 enge Becken, oder 95,5 pCt. 2 Frauen hatten normale Beeken; in 3 Fällen von Eolampsie ist über die Beckenverengung nichts im Geburtsbericht vermerkt.

Normales Becken . . . . . . . . . 2

Plattes Becken . . . . . . . . . . 10

Platt rhachitisches Becken . . . . . . 9

Allgemein verengtes Becken. .. . . . . 29

Allgemein verengt plattrhachitisches Becken 54

Allgemein and quer verengtes Becken . . 1

Spondylolisthetisches Becken . . . . . 1

Schräg verengtes Becken . . . . . . . 3

Nicht angegeben. . . . . . . . . . 3

Fragen wir nach der Conjugata vera der engen Becken, so finden wir unter den platten Becken Verengung I. Grades - 8,5: 2; II. Grades -7 excl.: 7; $1 \mathrm{Mal}$ wurde die Vera nicht angegeben.

Beim allgemein verengten Becken:

bis $8,5 \mathrm{~cm} \quad 2 \mathrm{mal}$

" $7 \mathrm{~cm} 13 \mathrm{mal}$

n $5,5 \mathrm{~cm} \quad 6 \mathrm{mal}$ (nicht vermerkt ist die Vera in 8 Fällen). Beim platt rhachitischen Becken:

bis $8,5 \mathrm{~cm} 1 \mathrm{mal}$

"7 $\mathrm{cm} 4 \mathrm{mal}$

" $5,5 \mathrm{~cm} 2 \mathrm{mal}$ ( 2 mal war die Vera nicht angegeben).

Beim allgemein verengt-platt rhachitischen Becken:

bis $8,5 \mathrm{~cm} 1 \mathrm{mal}$

n $7 \mathrm{~cm} 30 \mathrm{mal}$

" $5,5 \mathrm{~cm} 17 \mathrm{mal}$ (nicht angegeben in 6 Fällen).

Das allgemein und quer verengte Becken hatte eine Vera von $8 \mathrm{~cm}$, das spondylolisthetische von $7 \mathrm{~cm}$, zwei allgemein und schräg verengte Becken von $8 \mathrm{~cm}$ und $7 \frac{1}{2} \mathrm{~cm}$; ein durch Osteome der Beckenwand quer verengtes Becken eine Vera von $7 \underline{1 / 2} \mathrm{~cm}$.

Unter den 112 Frauen waren 64 Erstgebärende und 48 Mehrgebärende, oder $57,1 \mathrm{pCt}$. gegen $42,9 \mathrm{pCt}$.

Der Blasensprung erfolgte unter den 112 Fällen 55 mal vorzeitig ausserhalb der Klinik; in 16 Fällen vorzeitig hier in der Klinik; 
in 28 Fällen wurde die Fruchtblase künstlich gesprengt, in $3 \mathrm{Fällen}$ war es nicht möglich den Blasensprung der Zeit nach in Erfahrung zu bringen, in 10 Fällen erfolgte der Blasensprung zur rechten Zeit bei vollständig oder fast vollständig eröffnetem Muttermund.

100 Frauen waren im X. Monat schwanger, 8 im IX., 3 im VIII. und $1 \mathrm{im}$ VII. Monat. In 3 Fällen handelte es sich um Zwillinge.

Die Kindeslagen vertheilen sich in folgender Weise: $53 \mathrm{mal}$ wurde Schädellage I, 46 mal Schädellage II, 5 mal Stirnlage I, 5 mal Stirnlage II und 3 mal Gesichtslage (darunter 1 ungünstige) beobachtet.

In 67 Fällen stand der Kopf beweglich über dem Beckeneingang, in 21 Fällen fest im Beckeneingang, in 4 Fällen in Beckenmitte; 19 mal war der Kopf in Hinterscheitelbeineinstellung, $8 \mathrm{mal}$ in Vorderscheitelbeineinstellung eingestellt; in $10 \mathrm{Fällen}$ bandelte es sich um Nabelschnurvorfall, dabei in einem Falle um Nabelschnur- und Armvorfall; in 2 Fällen war der Arm vorgefallen, dabei $1 \mathrm{mal}$ bei festem Kopfe; $1 \mathrm{mal}$ war der rechte Fuss, $1 \mathrm{mal}$ eine Hand und ein Fuss vorgefallen.

Was die Muttermundsweite bei der Operation belangt, so finden wir 54 mal vollständigen Muttermund verzeichnet; in neun Fällen war er erst 3 markstückgross, in 10 Fällen 5 markstückgross, in 18 Fällen kleinhandtellergross, in 16 Fällen handtellergross. In 4 Fällen wurde er durch Incisionen erweitert; in einem Falle (2 Markstückgrösse) mit der Kornzange gedehnt; in einem Falle wurde bei 2 markstückgrossem Muttermund perforirt; in acht Fällen war er erst durch das Bossi'sche Instrument erweitert worden.

Die Frauen waren im Hause bis zur Operation:

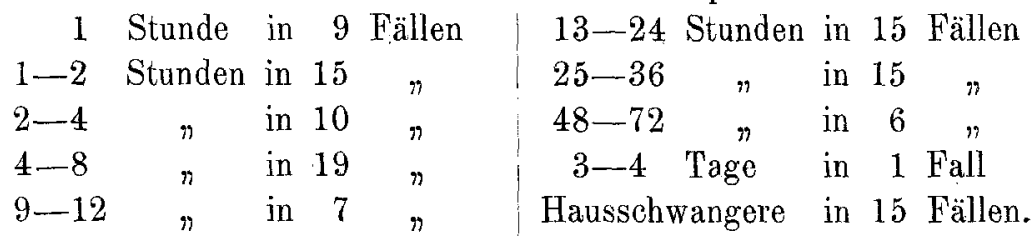

Warum wurde in den einzelnen Fällen das absterbende Kind perforirt? Wir wenden uns zunächst den 15 Fällen zu, die wir längere Zeit schon in der Schwangerschaft zu beobachten Gelegenheit hatten.

1. Fall (1892: 971). 32 jähr. II p. mit allgemein verengtem Becken. Vera $7-71 / 4$; I. partus. Sectio caesarea: lebender Knabe $50^{1 / 2} \mathrm{~cm}$, 
2990 g. Die Frau litt an sehwerer gonorrhoischer Kolpitis granulosa, weshalb man sich nicht von vornherein wieder zur Sectio entschliessen konnte. Die Blise war vorzeitig gesprungen; man wartete zunächst ab. Nach 9 stündigem Abwarten zeigte bei erst 2markstückgrossem Muttermunde das Kind deutliche Zeichen der Asphyxie, starke Verlangsamung der Herztöne und asphyctische Zuckungen. Zudem hatte sich wahrscheinlich, begünstigt durch die alte Uterusnaht, ein steigender Contractionsring ausgebildet, der die Beendigung der Entbindung anzeigte.

2. Fall (1893:1402): 30 jähr. IV p. mit allgemein verengt-plattrbachitischem Becken. Vera 81/4; Fruchtwasser vorzeitig ahgeflossen. Zur Vorbereitung der Weichtheile und Exziejung eines möglichst vollständigen Muttermundes, Erhaltung des noch übrigen Fruchtwassers: Kolpeurynter.

$\mathrm{Da}$ nach Entfernung des Kolpeurynters bei vollständigem Muttermund der Kopf nicht eintrat, ein Contractionsring sich ausgebildet hatte, und die schwankenden Herztöne immer bedenklicher wurden, so war bei dem hohen Kopfstand die Perforation der schonendste Eingriff. Heute käme die Hebotomie wohl in Frage.

In den vier folgenden Fällen (sämmtlich Erstgebärenden) war der Kopf bereits in das Becken eingetreten, einmal stand er bereits in Beckenmitte; die Gefahr der Kinder und Ausbildung eines Contractionsringes in 3 Fällen drängte zur Entbindung; trotz starken Zuges mit der Zange war der Kopt nicht tiefer getreten; man musste sich wohl oder übel zur Perforation entschliessen, da nach weiteren Tractionen das Kind auch abgestorben wäre. Ebenso in einem Falle (33 j. I p.) mit plattem Becken (Vera $8^{3 / 4}$ ) und fest in Beckenmitte stebender Gesichtslage, Kinn hinten, drohender Uterusruptur und sinkenden Herztönen.

Die folgenden 9 Fälle waren complicirt; theils durch Gonorrhoe oder Nephritis, theils durch Contractionsring, auftretendes Fieber der Mutter und schnelles Sinken der kindlichen Herztöne, sodass die Opferung des aussichtslosen kindlichen Lebens das Richtigste war.

In weiteren 12 Fällen kamen die Kreissenden nach erfolglosen Entbindungsversuchen vom Arzt geschickt zur Klinik. In 8 Fällen war die Zange, in 2 Fällen die Wendung, in 2 Fällen beides versucht worden. In 2 Fällen versuchte man hier noch, aber erfolglos, die hohe Zange; die Frauen kamen theils mit hohem Fieber, zersetztem Uterusinhalt, Contractionsring, vorgefallenem Arm oder Fuss hier an, theils mit Tetanus uteri. Eine Frau kam tamponirt mit Scheidenverletzungen nach hoher Zange und hohem Fieber und grosser Blasenscheidenfistel; dazu kam in allen Fällen Asphyxie der Kinder. Man hätte in diesen Fällen auch das lebensfrische Kind perforiren müssen.

In 2 Fällen musste man wegen schwerer Erkrankung der 
678 Meissuer, Perforation des lebensfrischen und absterbenden Kindes.

Mutter (Eklampsie und schwerer Nephritis) die künstliche Frühgeburt einleiten; das absterbende Kind wurde im Interesse der Mutter enthirnt.

In 10 Fällen war die Nabelschnur vorgefallen und das Kind im Absterben begriffen.

In 8 Fällen handelte es sich um Eklampsie und Sinken der kindlichen Herztöne.

In 12 Fällen hatte man vergeblich auf spontane Geburt gewartet und wollte, da die Beckenenge es zuliess, noch einen Wendungsversuch machen. Er unterblieb aber wegen dauernder Unregelmässigkeit und Sinken der kindlichen Herztöne.

In 9 Fällen waren der Perforation zur Rettung des absterbenden Kindes noch schonende Zangenversuche vorausgegangen, die zu keinem Erfolge führten.

In einem Falle bereitete man die Fran bereits zur Sectio caesarea vor. Sie war nach Untersuchung auf Anrathen eines Arztes mit bereits gesprungener Blase hierher geschickt worden. Man musste aber die Vorbereitungen aufgeben, da die Controle der Heratöne ein erlöschendes kindliches Leben feststellte.

Eine Erstgebärende kam bereits mit Fieber, eine nach $3 \mathrm{mal}$ 24 Stunden zurückliegendem Blasensprung, beide nach vielen inneren Untersuchungen durch Hebamme und Arzt. Das Kind im Absterben begriffen.

Die noch übrig bleibenden Fälle lassen sich in 2 Gruppen theilen: I. Frauen, die bereits zu spät in die Klinik eingeliefert wurden; II. solche, die nach längerer Beobachtung wegen eintretender Gefahr auch für die Mutter zur Perforation führen mussten.

I. In 2 Fällen (Erstgebärende) zwang Hinterscheitelbeineinstellung bei engem Becken neben Asphyxie des Kindes zur Perforation. Eine von diesen war trotz Untersuchungen in der Schwangerschaft und trotz des Rathes, bei Wehenbeginn sofort in die Klinik zu kommen, erst nach fast 2 tägiger Geburtsdauer mit fast vollständigem Muttermund und schwer asphyktischem Kinde erschienen. Die geplante beckenerweiternde Operation konnte nicht mehr vorgenommen werden. Die zweite, war bereits ausserbalb öfters innerlich untersucht worden, sodass man den Geburtscanal nicht mehr für aseptisch ansehen konnte. 4 Frauen mit engen Becken wurden nach langer Geburtsdauer mit Tympania uteri, fötidem Fruchtwasser und Fieber vom Arzt 
der Klinik überwiesen. Man hätte bei diesen Fällen keinen Augenblick zögern dürfen, auch das lebensfrische Kind im Interesse der Matter zu opfern.

Eine Erst- und eine IV. Gebärende mit engen Becken kamen mit Contractionsring und Hinterscheitelbeineinstellung in die Klinik; bei einer war das Fruchtwasser ca. 78 Stunden bereits abgeflossen; die Kinder absterbend. Es blieb keine andere Opcration als die Perforation übrig.

Weitere 2 Frauen wurden hereingeschickt mit Muttermundslippenödem, Contractionsring und absterbendem Kinde.

In 5 Fällen war wegen drohender Uterusruptur und Asphyxie der Kinder die Perforation die allein gegebene Operation.

In 2 Fällen hatte man bereits in der Schwangerschaft die Sectio caesarea bestimmt, einmal wegen Beckenverengerung durch Osteome der Beckenwand. Beide Erauen mussten aber nach vielen ausserhalb der Klinik erfolgten Untersuchungen als inficirt angesehen werden.

In allen diesen Fällen stand der kindliche Kopf im Missverhältniss zum Becken, beweglich über dem Beckeneingang, meist in ungünstiger Einstellung.

II. Fälle, die nach längerer Beobachtung, wegen eintretender Gefahr auch für die Mutter, zur Perforation führen mussten.

In 2 Fällen war bereits nach langer Geburtsdaner der Kopf bis in die Beckenmitte gerückt. Der Muttermund war jedoch nicht so erweitert, dass eine Zange das gefährdete Kind noch hätte retten können. In einem anderen Falle wurde der Muttermund mit dem Bossi'schen Instrument rasch erweitert; die allzu schwankenden Herztöne erlaubten jedoch nicht mehr die Zange.

In einem Falle trat Fieber der Mutter dazu.

In 3 Fällen handelte es sich um Contractionsring, beweglichen Kopf, noch nicht genügend eröffnete Weichteile und moribundes Kind. 5 mal um bewegliehen Kopf, Contractionsring und Fieber der Mutter. 3 mal um Hinterscheitelbeineinstellung, Fieber und Contractionsring, dazu hochgradige Erschöpfung der Frau. Bei einer Erstgebärenden mit spondylolisthetischem Becken trat ascendirendes Oedem der Weichtheile hinzu; man hatte von vornherein die Beckenverengerung unterschätzt und das Hereinragen der borizontalen Schambeinäste in das Becken verkannt. Es war dann bei dem absterbenden Kinde und der langen Geburtsdauer zur Sectio, caesarea zu spät geworden. 
680 Meissner, Perforation des lebensfrischen und absterbenden Kindes.

In 2 Fällen handelte es sich um $\mathrm{Z}$ willinge, deren 2. Kind perforirt werden musste. In dem einen Falle (26 j. II p.) hatte man den ersten in Kopfschieflage eingestellten Zwilling gewendet und Iebend extrahirt; der 2. Zwilling hatte sich in Gesichtslage eingestellt mit der Stirnnaht nahe der Symphyse; da die Herztöne zwischen 80 und 160 schwankten, beschloss man auch dieses Kind zu wenden; der Versuch missglückte wegen bereits ausgebildeten steigenden Contractionsringes; da beim Wendungsversuch die Nabelschnur pulslos gefühlt wurde, schloss man sofort die Perforation und Kranioklasie an. Im andern Falle fand sich bei der $25 \mathrm{j}$. I p. ein allgemein verengt platt-rhachitisches Becken. Als der Kopf des ersten Kindes im Beckenausgang war, trat ein Schwanken der Herztöne ein, weshalb man das Kind mit der Zange (noch lebend) entwickelte. Sofort nach Sprengen der 2. Fruchtblase trat ein Contractionsring auf, der die Wendung des 2. Kindes aus Kopflage mit Armvorfall misslingen liess. Das schwindende kindliche Leben und der Krampfring führten zur Perforation.

In den letzten 3 Fällen handelte es sich um Blutung 1 mal aus Placenta praevia bei einer $33 \mathrm{j}$. Erstgebärenden mit allgemein verengtem Becken und einer Vera von $8,5 \mathrm{~cm}$. Da man die Herztöne bereits im Galopprhytmus hörte und die Frau in Verblutungsgefahr schwebte (sie blutete seit 14 Tagen!), entschloss man sich rasch zur Perforation. Im andern Falle (19 j. I p.) rührte die Blutung mit grosser Wahrscheinlichkeit, aus vorzeitig gelöster Placenta her; der Kopf stand beweglich über dem Beckeneingang in II. Stirnlage. Die Herztöne schwankend. Da der Muttermund noch nicht genügend eröffnet war, schritt man zur Perforation. Im letaten Falle hatte sich in der hinteren Scheidenwand ein grosses Hämatom gebildet, aus dem es fortgesetzt blutete. Es handelte sich hier um eine 33 jähr. I para mit platt-rhachitischem Becken und einer Vera von $71 / 2 \mathrm{~cm}$. Die Blase war ausserhalb vorzeitig gesprungen. Da in der Blutung eine grosse Gefahr für die Mutter lag und das Kind im Absterben begriffen war, so wurde die Geburt durch Enthirnung und Extraction beendet.

Auch bei diesen 112 Geburten überwiegen die Knaben die Mädchen mit $69: 42=61,6$ pCt. : 37,5 pCt. In einem Falle war das Geschlecht nicht notirt.

Fragen wir nun nach den Erfolgen der Perforationen, die zur Rettung der Mütter stattfanden, so interessiren uns zunächst wieder die durch die Perforation und Kranioklasie gesetzten Ver- 
Meissner, Perforation des lebensfrischen und absterbenden Kindes. 681

letzungen der mütterlichen Weichtheile. In 4 Fällen wurde der nicht eröffnete Muttermund wegen Gefahr der Mutter incidirt; 2 mal bei Ekłanspie, $1 \mathrm{mal}$ wegen Fiebers, 1 mal wegen drohender Uterusruptur. Collumrisse wurden, auch die kleinste Einkerbung mitgerechnet, im Ganzen unter den 112 Geburten 27 aufgezeichnet gefunden oder $24 \mathrm{pCt}$. Die Perforation war dabei 6 mal bei vollständigem, $4 \mathrm{mal}$ bei handtellergrossem, 6 mal bei kleinhandtellergrossem, 6 mal bei 5 markstückgrossem, 4 mal bei 3 markstückgrossem, 1 mal erst bei 2 markstückgrossem Muttermunde ausgeführt worden. $11 \mathrm{pCt}$. Collumrisse erfolgten bei vollständigem, 44 pCt. bei 3 markstückgrossem, 60 pCt. bei 5 markstückgrossem, 33 pCt. bel kleinhandtellergrossem, 25 pCt. bei handtellergrossem Muttermunde. Es geht auch daraus wieder hervor, wie erwünscht auch bei der schädelzertrümmernden Operation die möglichst vollständige Erweiterung des Muttermundes ist. Verletzungen der äusseren Geschlechtstheile kamen in nennenswerther Weise nur 18 mal vor.

In 2 Fällen finden wir Blasenscheidenfisteln verzeichnet; bei denen die ausserhalb durch die Zange entstanden nicht mitgerechnet ist. In einem Falle war die Verletzung erfolgt durch die Operation bei kleinhandtellergrossem Muttermund, festem Kopf und rigiden Weichtheilen bei einer 33 jährigen Lrstgebärenden. Im zweiten Falle durch die sich nothwendig machende Anwendung des Kephalotrypters and der Boer'schen Knochenzange; man musste hierbei bei dreimarkstückgrossem Muttermund operiren; ein Collumriss war dabei nicht entstanden. Die Frau litt an schwerer Nephritis. Beide Fisteln hatten sich spontan in den ersten Tagen geschlossen. Die bereits ausserhalb erfolgte Blasenscheidenfistel wurde vernäht und heilte per primam.

Von den 112 Frauen machten 48 Frauen oder 42,8 pCt. ein vollkommen fieberfreies Wochenbett durch und konnten am 10. oder 11. Tage entlassen werden. 11 Frauen $=9,8$ pCt. hatten eine einmalige Steigerung, von 37,6 an gerechnet.

8 Frauen batten unruhige Curven durch hohen Puls, bedingt meistentheils durch Eklampsie, Atonia uteri, Placenta praevia. Nur kurze Zeit Fieber hatten 16 Frauen (Lochiometra, Gonorrhoe, belegter Riss, fötide Lochien). Länger als 5 Tage fieberten 17 Frauen: in Folge von fötiden Lochien, Portiobelag, Bronchitis, Gonorrhoe, Bronchopneumonie, Lochiometra oder parametraner Reizung. 
682 Meissner, Perforation des lebensfrischen und absterbenden Kindes.

Zehn Frauen konnten bis zum 18. Tag entlassen werden, 6 bis zum 28. Tage, 1 am 31. Tage. Von diesen 17 Frauen waren acht nach ausserhalb erfolgten Operationsversuchen oder nach vielen inneren Lntersuchungen inficirt in die Klinik gekommen.

3 Frauen hatten bereits Fieber in der Geburt; eine hatte Gonorrhoe, eine andere litt durch ungebührliches vorzeitiges Aufsitzen einige Tage lang an parametraner Reizung; in einem Falle lag Lochiometra vor; 1 Mal war wahrscheinlich durch den Zangenversuch bedingt ein Portiobelag entstanden; eine Fran hatte in der Geburt ascendirendes Oedem gehabt; eine Hausschwangere fieberte vom 3.-11. Tage wegen fötider Lochien; sie war nur 6 Stunden in der Geburt und hatte bereits nach den ersten Stunden 38,5 und Schüttelfröste (Gonorrhoe?).

Ein langdauerndes Wochenbett machten 5 Frauen durch; 1 Mal wegen Mastitis; $2 \mathrm{Mal}$ wegen Thrombose; $1 \mathrm{Mal}$ wegen Pelveoperitonitis; 1 Mal wegen parametranen Exsudates. Eine Frau mit Thrombose litt an florider Gonorrhoe; die an Pelveoperitonitis leidende kam nach ausserhalb am beweglichen Kopf erfolgtem Versuche mit der hohen Zange inficirt in die Klinik. Die wegen eines Exsudates lange fiebernde Frau kam ebenfalls nach 4 tägiger Geburtsdauer vom Arzt geschickt herein und war bereits als inficirt zu betrachten.

Von den 112 Frauen starben im Ganzen 7. Von ihnen müssen aber die ersten 6 Fälle (3 Eklampsien, 1 Caries der Wirbelsäule, I Narkose und 1 schwerste Nephritis) in Abzug gebracht werden, so dass nur 1 Todesfall der Perforation zur Last fällt $(112-6=106: 1=0,9$ pCt. Mortalität).

Zwei Frauen (Erstgebärende) starben am 2. Tage, ohne das Bewusstsein wiedererlangt $z u$ haben, im eklamptischen Coma. Hämorrhagien im Gehirn, eklamptische Lebernekrose stellte die Section fest in 1 Falle; im 2. wurde die Section verweigert. In einem 3. Falle von Wklampsie ergab die Section putride Pneumonie, eitrige Tracheitis und Meningitis, eitrigen Katarrh der Siebbeinhöhlen, Hirnödem, mässigen Milztumor. Die Frau war im Coma nach zahlreichen Anfällen bereits mit Lungenödem in die Klinik eingeliefert worden. Am 12. Tage trat der Tod ein. Die primäre Todesursache Aspirationspneumonie steht fest in dem Falle; der Frau ist per os in der Klinik nichts eingeflösst worden, so dass der Einwurf, die Schluckpneumonie habe sie in der Klinik bekommen, wegfällt. Eine 38 jährige IIIp. starb am 46. Wochen- 
bettstage an Pyämie in Folge von Caries der Wirbelsäule und Psoasabscess. Sie hatte bereits während der Geburt Fieber und fötiden Fluor am ersten Abend; in der Geburt bereits 38,4 und 92 Pulse; kurze Zeit nach der Geburt 40,5 und 132. Am 3. Tage traten Schüttelfröste ein, am 16. Tage trotz peinlichster Pflege Decubitus über dem linken Trochanter.

Eine Frau starb an den Folgen der Narkose 16 Stunden post oper. an plötzlich eingetretener Herzschwäche. Die Section ergab Glottisödem und ausgedehntes Oedem sämmtlicher Lungenlappen. Die Narkose war durch Erbrechen fäculenter Massen und durch eingetretene Asphyxie sehr gestört worden. Auch dieser Todesfall kann der Perforation nicht zugeschoben werden.

Ebensowenig der nächste, in welchem bei einer an schwerer Nephritis mit Amaurosis und Vitium cordis leidenden Ipara die Frühgeburt eingeleitet werden musste. Der Metreurynter lag stundenlang bei ihr erfolglos zur Erweiterung des Muttermundes. Schliesslich gelang sie durch Bossi, wonach die Geburt durch die Perforation des kleinen Kindes (8. Monat) schnell beendet werden konnte. Am 6. Tage Tod an schwerer Nephritis und puerperaler Sepsis. Wie sehr Nephritische zur Infection neigen, ist bekannt. $\mathrm{Zu}$ belasten wäre in diesem Falle vielleicht der Metreurynter, sicherlich nicht die Perforation.

So bleibt nur der letzte Fall übrig, in welchem der Tod am 11. Tage des Wochenbettes an Streptokokken-Endometritis erfolgte. Die Geburt hatte bis zur Operation 1 Tag 23 Stunden gedauert und ging unter den günstigsten Bedingungen, bei vollständigem Muttermunde, durch Enthirnung des absterbenden Kindes vor sich. Es kann keinem Zweifel unterliegen, dass diesen Todesfall die Perforation i. e. die klinische Therapie versehuldet.

Fassen wir die Gesammtsterblichkeit im I. und II. Theil zusammen, so kamen unter 169 Perforationen des lebensfrischen bezw. absterbenden Kindes, welche in den letzten $14^{1} / 2$ Jahren. zur Ausführung kamen, 9 mütterliche Todesfälle vor, von denen aber nur $2(162: 2)=1,2$ pCt. der Perforation und Kranioklasie zugeschrieben werden können.

Was haben wir nun zu thun, um die Perforation des. lebenden Kindes auf ein Minimum herabzudrücken? Es muss vor Allem hervorgehoben werden, dass in einigen unserer Fälle das allzu lange Abwarten auf einen Spontanpartus 
684 Meissner, Perforation des lebensfrisehen und absterbenden Kindes.

die Schuld daran trug, dass im Interesse der Mutter das lebende Kind geopfert werden musste. Ebenso führte in einzelnen Fällen das lange Abwarten zu dem Absterben des Kindes. Handelt es sich z. B. bei Erstgebärenden um ein stärkeres Missverbältniss zwischen Becken und kindlichem Kopfe, stellt sich der Kopf zwar günstig ein, configurirt sich aber bei kräftigen Wehen und genügend eröffneten Weichtheilen nicht, was sich wohl nach ungefähr 6 stündiger Geburtsarbeit beurtheilen lässt, so schreite man nach dieser Abwartezeit zur Becken-erweiternden Operation, und versuche nicht erst die hohe Zange. Hat sich der Kopf ungünstig, z. B. in ausgesprochener Hinterscheitelbeineinstellung bei einer Ipara eingestellt und wird die Stellung nicht in den ersten Stunden corrigirt, so schreite man in solchen Zwischenfällen bei noch aseptischem Genitaltractus zur Sectio caesarea auch aus relativer Indication. Die Erfahrung lehrt, dass selbst nach Hebotomie ein in ausgesprochener Hinterscheitelbeineinstellung befindlicher Kopf der Zange nicht folgte und schliesslich angebohrt werden musste.

Ebenso verfahre man bei Mehrgebärenden, wo die früheren Geburten mit todten Kindern durch Perforation endeten, und schreite, vorausgesetzt, dass die Vorbedingungen erfüllt sind, zur Sectio caesarea oder Hebotomie mit entsprechender Geburtsbeendigung durch Zange oder Wendung und Extraction je nach dem Stande des Kopfes.

Das allzulange Abwarten war auch in einigen dieser Fälle von Nachtheil gewesen, indem sich ein Contractionsring ausbildete oder das kindliche Leben in Gefahr kam, so dass dann immer als Erstes die Frage zu beantworten galt: "Was ist nunmehr das Schonungsvollste für die Mutter?"

Anders liegen aber auch heute noch die Verhältnisse und die Maassnahmen beim engen Becken für den praktischen Geburtshelfer. Die Kliniken haben die Verpflichtung, ihn nicht im Stiche zu lassen. So sollen ihm vielmehr ganz klare Directiven geben, wie er sich zu verhalten hat, wenn sich Sectio caesarea bezw. Hebotomie als unmöglich bezw. unausführbar berausstellen und er nun vor einem beliebigen, in die Gruppe unserer jetzigen Besprechung gehörenden Fall gestellt wird.

Zunächst bleibt immer der Rath beherzigenswerth, dass Frauen mit engem Becken, namentlich nach früheren schweren Operationen, bei erneut eintretender Schwangerschaft sich in einer Klinik An- 
weisung geben lassen sollen, ob es aussichtsvoll ist, in der 35 . bis 36. Woche die Frühgeburt einleiten zu lassen oder richtiger, bis zum Fnde der Zeit zu warten.

Sicherlich giebt es für die mässigen Beckenverengerungen von $8,5-7,75 \mathrm{~cm}$ Vera eine Zahl von Fällen, wo unter Befolgung aller Cautelen, bei guter Technik, bei correcter Indicationsstellung durch die künstliche Frühgeburt lebende Kinder erzielt werden können, die, wie andere Neugeborene, volle Aussicht haben, am Leben erhalten zu bleiben (s. Leopold und Konrád, Zur Lebensberechtigung der künstlichen Frühgeburt, dieses Heft).

Am Ende der Schwangerschaft aber wird der praktische Geburtshelfer, sobald er je nach den räumlichen, örtlichen und häuslichen Verhältnissen die Unmöglichkeit anerkennen muss, die Gebärende in eine Klinik zu verweisen, unter allen Umständen auch heute noch nach dem Grundsatz zu handeln haben: "Erst die Mutter, dann das Kind!"

Natürlich wird kein Einsichtiger hier herauslesen wollen, dass das Kind nicht das gleiche Anrecht an Lebenserhaltung habe, wie die Mutter. Auch der praktische Geburtshelfer hat die Verpflichtung, die Errungenschaften der Klinik in das praktische Leben zu übertragen und Kaiserschnitt und Hebotomie auszuführen, wenn der ganze Fall es zulässt, aber nie auf Kosten der Mutter.

Dieses ihr Leben ist ein Heiligthum, welches ja ein Kind erst entstehen und dann zur Welt kommen lässt. Dieses Heiligthum bedarf des höchsten Schutzes.

Im Zweifelsfalle und bei schwankender Wagschale wird der praktische Geburtshelfer, sollte er ganz auf sich allein angewiesen sein, auf noch lange Zeit hinaus mit der Perforation selbst des lebenden Kindes mehr Erfolg für die Menschheit haben als ohne sie. 